\title{
Mineralization of Aqueous Benzenesulfonates by Contact Glow Discharge Electrolysis
}

\author{
Ryo Amano, Shunsuke TOMIZAWA, and Meguru TEZUKA*
}

\begin{abstract}
Department of Material Science and Engineering, Graduate School of Engineering, Saitama Institute of Technology (1690 Fusaiji, Okabe, Saitama 369-0293, Japan)
\end{abstract}

Received June 28, 2004 ; Accepted September 1, 2004

\begin{abstract}
Contact glow discharge electrolysis(CGDE) of benzenesulfonates in a neutral phosphate buffer solution was investigated. Benzenesulfonic acid was smoothly degraded and eventually mineralized to inorganic carbon and sulfate ion. As the intermediate products, some phenolic compounds were detected as well as carboxylic acids such as oxalate and formate. Also in CGDE of p-toluenesulfonic acid, quite similar results were obtained. Based on the products and kinetic consideration, it was assumed that hydroxyl radicals would play a crucial role in the oxidative degradation of aqueous benzenesulfonates.
\end{abstract}

Key Words : Mineralization, Glow Discharge Electrolysis, Benzenesulfonates, Hydroxyl Radical

\section{Introduction}

In these decades, the environmental problems caused by harmful chemicals which might remain and biologically accumulate in nature have been globally of serious interest. As the sources of these pollutants, the drainage from factories as well as household has been especially emphasized. Thus, the techniques called AOPs (Advanced Oxidation Processes) have been developed in the field of the treatment of waste water. "In order to destroy a diversity of troublesome organics effectively, there are mostly used ozone and hydrogen peroxide as potent oxidants under the irradiation of energy such as UV, ultrasonic wave, electron beam and so on. The catalysis of Fenton reagent or $\mathrm{TiO}_{2}$ is often a helpful option in AOPs.

Of late, the application of plasmas to waste treatment is a matter of growing concern. We have paid special attention to "contact glow discharge electrolysis (CGDE)" ${ }^{-4)}$, which resembles a conventional electrolysis apart from that a continuous gaseous plasma can be sustained between the solution surface and the electrode in contact with it. Actually, we reported that CGDE could be successfully carried out for the aqueous solution containing organic compound such as phenols, ${ }^{5}$ chlorophenols, ${ }^{6)}$ aniline ${ }^{7)}$ and benzoic acid ${ }^{8)}$ to convert them into inorganics efficiently. On the whole, CDGE features rapid and indiscriminate mineralization of organic compounds relative to the above AOPs.

In the present study, benzenesulfonic acid(BS) and ptoluenesulfonic acid(TS) were subject to CGDE. In a sense, both might be regarded as model compounds of linear alkyl benzenesulfonic acid (LAS) which is a wellknown surfactant and one of principal ingredients in synthetic detergent. When LAS was once drained as domestic sewage it brought about a critical social issue. The pollution of rivers or lakes was widespread in human liv- ing areas, because LAS was an artificial chemical of mass production and quite persistent in the environment. The most of surfactants have sulfo groups to fulfill the sufficient hydrophilicity and solubility even under uncommon conditions. Therefore, it seems significant to collect the mechanistic knowledge about degradation of aqueous benzenesulfonates by means of CGDE.

\section{Experimental}

The electrolytic solution was prepared by dissolving benzenesulfonic or p-toluenesulfonic acid in phosphate $\mathrm{pH}$ standard solution $\left(8.695 \mathrm{mM}\left(\mathrm{M}=\mathrm{mol} \mathrm{dm}^{-3}\right)\right.$ potassium dihydrogenphosphate and $30.43 \mathrm{mM}$ disodium hydrogenphosphate, $\mathrm{pH}$ 7.4). The reaction apparatus and assembly are schematically illustrated in Fig.1. A cylindrical cell was used. The anode, from which the discharge was emitted, was a pointed platinum wire $(0.8$ $\mathrm{mm}$ diameter) sealed in a glass tube which was introduced into the cell. The cathode of a stainless steel plate was held in another glass tube, the bottom of which was equipped with a glass frit, and immersed into the solution. The cell was placed in an ice-water bath. The anode of Pt wire was dipped into the solution by the depth of ca. $1 \mathrm{~mm}$. To initiate CGDE, high voltage, usually $500 \mathrm{~V}$, was supplied from a DC source. The electrolytic current was managed to average approximately $70 \mathrm{~mA}$ by adjusting the depth of the anode. The quantity of electricity passed during the electrolysis was measured with a digital coulomb-meter. In the course of the reaction, the solution was gently stirred with a magnet bar. A small portion of solution was periodically sampled out and analyzed by HPLC. In order to determine the intermediate products as well as recovered starting materials, Inertsil ODS-3 V was employed as a column packing. In addition, Shodex Ionpak KC-811 and HAMILTON PRP-X100 were used for analyses of carboxylic acids and sulfate ion, re- 


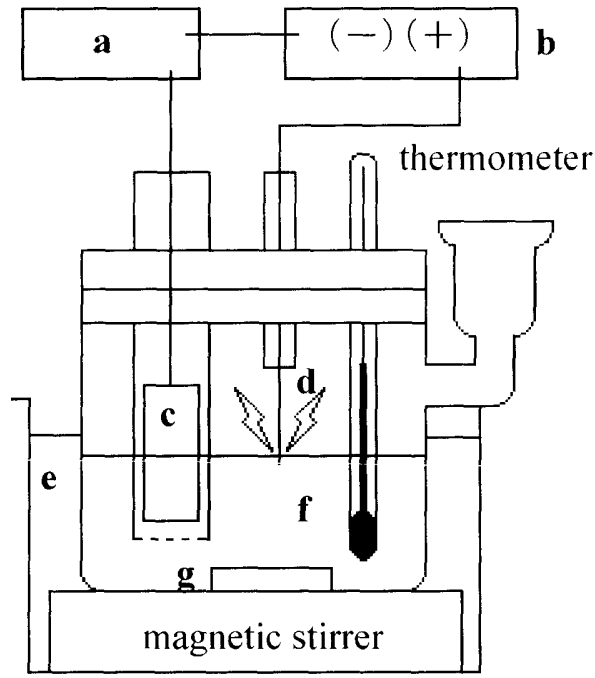

Fig. 1 Apparatus for CGDE. a:coulomb-meter; b:DC source; c:cathode; d:anode; e:ice-water bath; f:electrolytic solution; g: Teflon-coated magnet bar.

spectively. The concentration of total organic carbon (TOC) in the electrolytic solution was estimated by a TOC analyzer. Identification of products were occasionally confirmed by means of mass spectrometry.

\section{Results and Discussion}

The degradation of benzenesulfonic acid (BS) proceeded smoothly when CGDE was carried out on the aqueous solution containing it, as seen in Fig. 2. The decay curve of TOC are also displayed, where the initial concentration of BS $\left(\mathrm{C}_{1}\right)$ was $5.34 \mathrm{mM}$. Note that this concentration of BS corresponds to $385 \mathrm{ppm}$ of carbon concentration. On starting the reaction, the concentration of BS immediately decreased with the discharge time. After $180 \mathrm{~min}$, BS was completely extinguished. Disappearance of BS was followed by the monotonous reduction of TOC. At $240 \mathrm{~min}$ of discharge time, the decline of TOC amounted to $90 \%$. This means that BS could be readily converted to inorganic carbon(IC), which might exist as hydrogencarbonate in the solution or might be released as carbon dioxide to the gas phase. In any case, they are the end products in the mineralization of organic carbon. The variation in the concentration of sulfate ion as another inorganic product from BS is exhibited in Fig.2, too. It increased promptly until $120 \mathrm{~min}$ of discharge time and became gradually saturated afterwards. These results demonstrate that BS dissolved in an aqueous solution can be oxidatively decomposed and ultimately mineralized by means of CGDE.

The yields of both final products, namely IC and sulfate, are tabulated as well as the conversion of BS in Table 1. There are also presented the yields of two major intermediates, oxalate and formate. The yield is defined as moles of a certain element $(\mathrm{C}$ or $\mathrm{S})$ in each product per moles of that in BS reacted. The conversion of BS went up to unity with the increasing discharge time. On the other hand, the yield of sulfate was within 0.8-0.9 in the earlier stage of the run, while the value finally

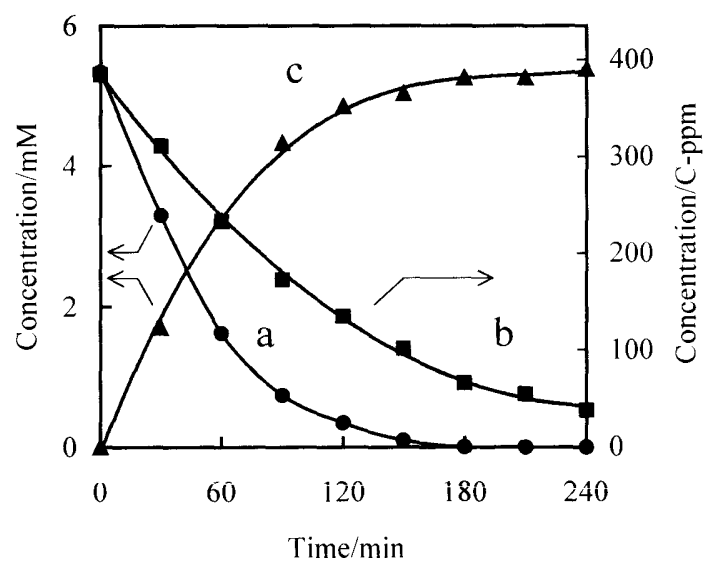

Fig. 2 CGDE of BS in phosphate buffer solution ( $\mathrm{pH} 7.4)$. Initial concentration of BS : $5.34 \mathrm{mM}$, (a) BS, (b) TOC, (c) sulfate ion.

Table 1 The yields of products in CGDE of BS.a)

\begin{tabular}{|c|c|c|c|c|c|c|c|}
\hline \multirow{3}{*}{$\begin{array}{c}\text { Time/ } \\
\min \end{array}$} & \multirow{3}{*}{$\begin{array}{c}\text { BS } \\
\text { conversion }\end{array}$} & \multicolumn{6}{|c|}{ Product yield } \\
\hline & & \multicolumn{2}{|c|}{ Sulfur-based } & \multicolumn{4}{|c|}{ Carbon-based } \\
\hline & & Sulfate & $\mathrm{Nd}^{\mathrm{b})}$ & Oxalate & Formate & IC & $\mathrm{Nd}^{\mathrm{b})}$ \\
\hline 30 & 0.38 & 0.83 & 0.17 & 0.29 & 0.03 & 0.50 & 0.18 \\
\hline 60 & 0.70 & 0.87 & 0.13 & 0.25 & 0.03 & 0.57 & 0.15 \\
\hline 90 & 0.80 & 0.94 & 0.06 & 0.21 & 0.02 & 0.64 & 0.13 \\
\hline 120 & 0.94 & 0.97 & 0.03 & 0.20 & 0.02 & 0.69 & 0.09 \\
\hline 150 & 0.98 & 0.96 & 0.04 & 0.16 & 0.02 & 0.73 & 0.09 \\
\hline 180 & 1.00 & 0.99 & 0.01 & 0.13 & 0.02 & 0.83 & 0.02 \\
\hline 210 & 1.00 & 0.99 & 0.01 & 0.10 & 0.02 & 0.86 & 0.02 \\
\hline 240 & 1.00 & 1.00 & 0 & 0.07 & 0.02 & 0.90 & 0.01 \\
\hline
\end{tabular}

a) Initial concentration of BS : $5.34 \mathrm{mM}$, b) Not determined

reached unity, suggesting that the sulfur-containing organic substances should be intermediately derived in a sequence of collapse of BS. As denoted by sulfur-based $\mathrm{Nd}$ in Table 1, most of them have not been welldetermined yet. A few exceptions are p-phenolsulfonic acid and diphenylsulfone. Anyhow, all of them were ultimately oxidized to liberate sulfate ion. Indeed, sulfite ion could not be found in an appreciable amount. Neither was detected peroxodisulfate ion, which had been reported as a negligible product in CGDE of a dilute sulfuric acid solution without organic compounds. ${ }^{9,10)}$

A similar tendency was observed as to the carbonbased product yields. At the final stage, the yield of IC became prevailing. However, various organic byproducts rose and fell during the run. The most major of them were oxalate and formate. Phenolic compounds such as phenol, pyrocatechol, hydroquinone, 1,2,3 trihydroxybenzene, and 1,2,4-trihydroxybenzene were detected in addition to the sulfur-containing ones mentioned above, although the yields of these products were far below 0.01 , respectively. These hydroxyphenols were intermediately produced in CGDE of phenol, too, and were reasonably explained to result from the successive attacks of hydroxyl radicals onto benzene nucleus prior to the ring cleavage. ${ }^{5)}$ The yields of oxalate and formate showed an analogous variation with the discharge time, whereas the former was about ten times higher. The fact 
that they were partly left intact even in the end of run indicates that both of them might be comparably durable to CGDE. Furthermore, it seems improbable that they may be generated in series, since no formate was practically given in the reaction of oxalate as a starting material. ${ }^{\prime \prime \prime}$ In contrast, phenolic by-products were much more susceptible intermediates and, in consequence, became inconspicuous in the later stage of CGDE.

The effective mineralization similarly progressed when the solution containing p-toluenesulfonic acid(TS) was subject to CGDE. The variations in the concentrations of TS and sulfate ion formed werc esscntially same as those of BS and sulfate in Fig. 2, when the initial concentration of TS was about $5 \mathrm{mM}$. Around the discharge time of 150 minutes, $100 \%$ of TS disappeared and subsequently sulfate ion led to the quantitative concentration. The decay of TOC was also identical with that in CGDE of BS. Oxalate and formate were again major byproducts. The additional intermediates were remarkable in the beginning of reaction. Expectedly, p-cresol and methylcatechols were obtained in place of phenol and pyrocatechol from BS, respectively. Methylhydroquinone was not formed, presumably due to the steric hindrance brought by the methyl group in TS, while p-toluquinol(4hydroxy-4-methyl-2,5-cyclohexadien-1-one) was detected as a possible alternative. In comparison with BS, more complicated mixture of intermediates resulted. However, the methyl group itself appears relatively resistant to the oxidation induced by CGDE, since no aromatic products originating from its oxidation were substantially obtained.

The decay curve of BS in Fig.2 looks exponential. In practice, a linear relationship was depicted with good correlation when the data was plotted according to the integral formula for the first-order rate law. This is also the case for TS. Generally, when CGDE is carried out on aqueous solution containing relatively low concentration of organics, the disappearance obeys the first-order rate law, $,^{5,7}, 8$ and it has been rationalized by the concept that the attack of hydroxyl radicals toward the starting materials should be a rate-limiting step in the over-all reactions. ${ }^{12.13)}$ Therefore, one might conclude that hydroxyl radicals would still act as a key-species to accomplish the mineralization in CGDE of aqueous benzenesulfonates.

In order to further elucidate the reaction pathway, the elaborate attempts to enable the detailed analyses of intermediate products and reaction kinetics are going under way.

\section{Acknowledgement}

Financial support from High-Tech Research Center of Saitama Institute of Technology is acknowledged.

\section{References}

1) Saishin Koudo Mizushori Gijutsu, NTS, Tokyo, 197 (1999) (in Japanese).

2) A. Hickling and M. D. Ingram, J. Electroanal. Chem., 8, 65 (1964).

3) A. Hickling and M. D. Ingram, Trans. Faraday Soc., 60, 783 (1964).

4) K. Harada and T. Iwasaki, Nature, 250, 426 (1974).

5) M. Tezuka and M. Iwasaki, Denki Kagaku (presently Electrochemistry), 65, 1057 (1997).

6) M. Tezuka and M. Iwasaki, Thin Solid Films, 316, 123 (1998).

7) M. Tezuka and M. Iwasaki, Thin Solid Films, 386, 204 (2001).

8) M. Tezuka and M. Iwasaki, Plasmas \& Ions, 1, 23 (1999).

9) Y. Kanzaki, N. Nishimura, J. Toba, M. Konuma, and O. Matsumoto, Denki Kagaku (presently Electrochemistry), 52, 126 (1984).

10) M. Tezuka, Denki Kagaku (presently Electrochemistry), 61, 794 (1993).

11) To be submitted elsewhere.

12) I. Somiya and H. Yamada, EISEI KAGAKU, 33, 365 (1987) (in Japanese).

13) B. Boye, M. M. Dien, and E. Brillas, Environ. Sci. Technol., 36, 3030 (2002). 